\title{
The problem of Euler/Tarry revisited
}

\section{Dieter Betten ${ }^{1}$}

Received: 4 June 2020 / Accepted: 1 November 2020 / Published online: 23 November 2020

() The Author(s) 2020

\begin{abstract}
The problem of Euler/Tarry concerning 36 officers can be formulated in mathematical terms: Can a latin square of order 6 have an orthogonal square, or equivalently, are there 6 pairwise disjoint transversals? This was first answered (in the negative) by Tarry (1900/01). We prove the following Theorem: If a latin square of order 6 admits a reflection, i. e. an automorphism of order two which fixes the main diagonal elementwise, then it has no orthogonal square. We list the 12 isomorphism types of latin squares of order 6 and see: they all admit such a reflection. So we get a solution of the Euler problem without the tedious task of tracing the transversals.
\end{abstract}

Keywords Orthogonal arrays · Latin squares - Room squares · Orthogonal designs · configurations

\section{Recalling some notions}

We recall Euler's 36 officers Problem: Can 36 officers, drawn from 6 different ranks and also from 6 different regiments, be ranged in a square so that in each line (both horizontal and vertical) there are 6 officers of different rank and different regiments?

This was a famous problem at Euler's time, and Euler wrote a paper concerning it, Euler (1782). The first proof of the non-existence was by Tarry (1901), so we may call this problem now the Euler/Tarry problem. The problem can be formulated in mathematical terms by the existence of a MOL(6) (a pair of orthogonal latin squares LQ(6) of order 6), Denes and Keedwell (1974). Further proofs for the non-existence were given, also very short ones, (Betten 1983; Beth et al. 1985; Stinson 1984).

We give an example (Fig. 1): In each cell of the $(6 \times 6)$-array, there is a pair of numbers. We call the first number the digit (corresponding to the rank) and the second number the transversal (corresponding to the regiment).

$\varangle$ Dieter Betten

reteid@math.uni-kiel.de

1 Christian-Albrechts-University Kiel Department of Mathematics, Ludewig-Meyn-Str. 4, 24118 Kiel, Germany 
Fig. 1 Example of a MOL(6)

$\begin{array}{llllll}25 & 46 & 52 & 64 & 11 & 33 \\ 16 & 54 & 61 & 35 & 23 & 42 \\ 51 & 63 & 45 & 26 & 32 & 14 \\ 62 & 15 & 36 & 53 & 44 & 21 \\ 43 & 31 & 24 & 12 & 56 & 65 \\ 34 & 22 & 13 & 41 & 6 ? & 5 ?\end{array}$

Fig. 2 Incidence structure of the MOL(6)

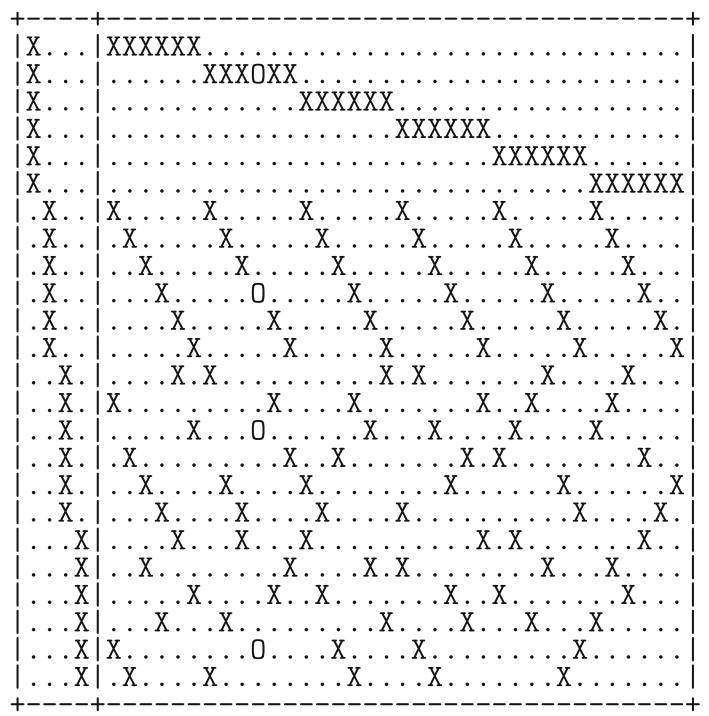

We can describe this as an incidence structure on 24 variables with 4 subsets of order 6 (the 6-blocks) and 36 subsets of order 4 (the 4-blocks), Fig. 2. The four 6blocks are $R=\left\{r_{1}, r_{2}, \ldots, r_{6}\right\}$ (the six rows), $C=\left\{c_{1}, c_{2}, \ldots, c_{6}\right\}$ the six columns, $D=\left\{d_{1}, d_{2}, \ldots, d_{6}\right\}$ (the six digits) and $T=\left\{t_{1}, t_{2}, \ldots, t_{6}\right\}$ (the six transversals). Each 4-block has the form $(r, c, d, t), r \in R, c \in C, d \in D, t \in T$. The main condition is: each pair of distinct variables in on exactly one block.

In the last line of the first figure we had to put two question marks since a MOL(6) does not exist. We also marked a special 4-block $(r, c, d, t)=(2,4,3,5)$. This can be seen in Fig. 2 as the 4-block with $\mathrm{O}$ instead of X.

In the standard notation (Fig. 1) the sets of rows $\mathrm{R}$ and columns $\mathrm{C}$ are distinguished: they define the $(6 \times 6)$-array, where the 36 pairs $(d, t) \in D \times T$ are inserted. But in the notation of Fig. 2 (linear space on 24 variables) none of the four sets $R$ (first 6-tuple), $C$ (second 6-tuple), $D$ (third 6-tuple) and $T$ (fourth 6-tuple) is preferred. Here one can also see the four latin squares of order 6 , which are contained in the $\operatorname{MOL}(6)$ : these are the restrictions to the sets $R \cup C \cup D, R \cup C \cup T, R \cup D \cup T$ and $C \cup D \cup T$.

The notion of isomorphism:

We call two latin squares $V_{1}=R_{1} \cup C_{1} \cup D_{1}$ and $V_{2}=R_{2} \cup C_{2} \cup D_{2}$ isomorphic if there is a bijection $\varphi: V_{1} \rightarrow V_{2}$ which maps blocks to blocks. If $\varphi$ maps rows to rows, columns to columns and digits to digits, we get 22 types, called the isotopy 
types in Colbourne and Dinitz (1996). But if in the general case, the map $\varphi$ is allowed to permute the three sets $R, C$ and $D$, then the number of types is 12 . These are the isomorphism types as linear spaces, called the main types in Colbourne and Dinitz (1996).

\section{The 12 latin squares LQ(6) of order 6}

For each pair of rows of a given latin square of order 6 there are four permutations possible. We describe the permutations by their cycle distribution and abbreviate these by $6,2,4$ and 3 :

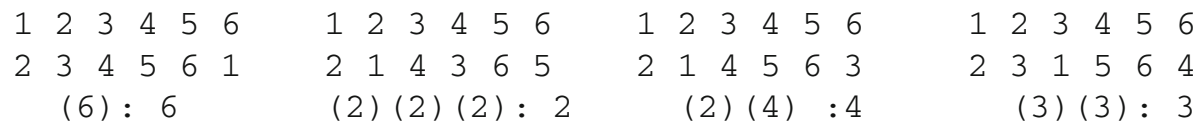

In Betten (1984) we proved that each LQ(6) has up to isomorphism a pair of type 3, and using this, we gave a quick classification of the 12 isomorphism types of latin squares LQ(6). These 12 squares also occur in Betten and Betten (2001) where we classified linear spaces with 18 points. In the following list we give the 12 types of $\mathrm{LQ}(6)$ together with some invariants.

Definition of $\mu_{3}$ : A latin square LQ $(6)$ is defined on a $(6 \times 6)$-array having 6 rows $\mathrm{r}$ and 6 columns c. On each position $(r, c), r, c \in\{1,2,3,4,5,6\}$ there is given a digit $d \in\{1,2,3,4,5,6\}$. So the latin square corresponds to a 36-element subset $L$ of the 3-dimensional cube $\{(r, c, d), r, c, d \in\{1,2,3,4,5,6\}\}$ with edge length 6 and $6^{3}=216$ elements. These 36 elements must be chosen suitably so that the conditions for a latin square are fulfilled. We now consider subcubes with edge length 3 , each defined by a triple $1 \leq r_{1}<r_{2}<r_{3} \leq 6$ of rows, a triple $1 \leq c_{1}<c_{2}<c_{3} \leq 6$ of columns, and a triple $1 \leq d_{1}<d_{2}<d_{3} \leq 6$ of digits. Let $\Sigma$ be the set of these subcubes, $|\Sigma|=\left(\begin{array}{l}6 \\ 3\end{array}\right)^{3}=8000$. For each $W \in \Sigma$ we determine the number $|W \cap L|$, i.e., the number of elements of the latin square which are contained in W. We now set

$$
\mu_{3}=\max \{|W \cap L|, W \in \Sigma\}
$$

The first 4 squares have $\mu_{3}=9$. The squares no 5-7 have $\mu_{3}=8$ and are derived from numbers 1 to 3 by rotating the 4 central elements by $90^{\circ}$. No. $7-12$ have $\mu_{3}=7$.

We define $\lambda$ as the number of sub-LQ(2), and $\tau$ is the number of transversals.

Then all permutations of pairs of rows, pairs of columns and pairs of digits are given. Let $A$ be the automorphism group and $I$ the inner group (which maps rows to rows, columns to columns and digits to digits) then we give the orders $|A|$, and $|A / I|$ and the type of the quotient group $A / I$ which permutes the three dimensions (rows, columns and digits). We give also the numbers in the list of Tarry and in the list of Fisher Yates. 
The 12 isomorphism types of the LQ(6)

1) \begin{tabular}{lll|lll}
3 & 1 & 2 & 6 & 4 & 5 \\
\hline 5 & 4 & 6 & 2 & 1 & 3 \\
4 & 6 & 5 & 1 & 3 & 2 \\
6 & 5 & 4 & 3 & 2 & 1 \\
\hline
\end{tabular}

5) \begin{tabular}{ccc|ccc|}
\hline 1 & 2 & 3 & 4 & 5 & 6 \\
2 & 3 & 1 & 5 & 6 & 4 \\
3 & 1 & $\mid \overline{6}$ & $\overline{2} \mid$ & 4 & 5 \\
\hline 5 & 4 & $\mid \underline{2}$ & $\underline{6} \mid$ & 1 & 3 \\
4 & 6 & 5 & 1 & 3 & 2 \\
6 & 5 & 4 & 3 & 2 & 1 \\
\hline
\end{tabular}

2) \begin{tabular}{lll|lll|}
\hline 1 & 2 & 3 & 4 & 5 & 6 \\
3 & 1 & 2 & 5 & 6 & 4 \\
2 & 3 & 1 & 6 & 4 & 5 \\
\hline 5 & 4 & 6 & 1 & 3 & 2 \\
4 & 6 & 5 & 2 & 1 & 3 \\
6 & 5 & 4 & 3 & 2 & 1 \\
\hline
\end{tabular}

$3)$\begin{tabular}{|lll|lll|}
\hline 1 & 2 & 3 & 4 & 5 & 6 \\
3 & 1 & 2 & 6 & 4 & 5 \\
2 & 3 & 1 & 5 & 6 & 4 \\
\hline 4 & 6 & 5 & 1 & 2 & 3 \\
5 & 4 & 6 & 3 & 1 & 2 \\
6 & 5 & 4 & 2 & 3 & 1 \\
\hline
\end{tabular}

4) \begin{tabular}{lll|lll|}
\hline 3 & 1 & 2 & 4 & 5 & 6 \\
1 & 2 & 3 & 6 & 4 & 5 \\
2 & 3 & 1 & 5 & 6 & 4 \\
\hline 4 & 5 & 6 & 1 & 2 & 3 \\
6 & 4 & 5 & 2 & 3 & 1 \\
5 & 6 & 4 & 3 & 1 & 2 \\
\hline
\end{tabular}
6) \begin{tabular}{|ccc|ccc|}
\hline 1 & 2 & 3 & 4 & 5 & 6 \\
3 & 1 & 2 & 5 & 6 & 4 \\
2 & 3 & $\mid \overline{6}$ & $\overline{1} \mid$ & 4 & 5 \\
\hline 5 & 4 & $\mid \underline{1}$ & $\underline{6} \mid$ & 3 & 2 \\
4 & 6 & 5 & 2 & 1 & 3 \\
6 & 5 & 4 & 3 & 2 & 1 \\
\hline
\end{tabular}

7) \begin{tabular}{|ccc|ccc|}
\hline 1 & 2 & 3 & 4 & 5 & 6 \\
3 & 1 & 2 & 6 & 4 & 5 \\
2 & 3 & $\mid \overline{5}$ & $\overline{1} \mid$ & 6 & 4 \\
\hline 4 & 6 & $\mid \underline{1}$ & $\underline{5} \mid$ & 2 & 3 \\
5 & 4 & 6 & 3 & 1 & 2 \\
6 & 5 & 4 & 2 & 3 & 1 \\
\hline
\end{tabular}

8) \begin{tabular}{llllll|}
\hline (1) & 2 & 3 & 4 & 5 & 6 \\
2 & $(1)$ & 6 & 5 & 3 & 4 \\
3 & 6 & $\mathbb{1}$ & 2 & 4 & 5 \\
4 & 5 & 2 & $\mathbb{1}$ & 6 & 3 \\
5 & 3 & 4 & 6 & $\mathbb{1}$ & 2 \\
6 & 4 & 5 & 3 & 2 & $\mathbb{1}$ \\
\hline
\end{tabular}

$10)\left(\begin{array}{ll|llll|}\hline 2 & 1 & 3 & 4 & 5 & 6 \\ 1 & 2 & 4 & 3 & 6 & 5 \\ \hline 3 & 5 & 2 & 6 & 4 & 1 \\ 5 & 3 & 6 & 1 & 2 & 4 \\ 4 & 6 & 5 & 2 & 1 & 3 \\ 6 & 4 & 1 & 5 & 3 & 2 \\ \hline\end{array}\right.$

11)

\begin{tabular}{|cc|cccc|}
\hline$(2)$ & 1 & 3 & 5 & 4 & 6 \\
1 & $(2)$ & 6 & 4 & 3 & 5 \\
\hline 3 & 6 & 1 & 2 & 5 & 4 \\
5 & 4 & 2 & 1 & 6 & 3 \\
4 & 3 & 5 & 6 & $(1)$ & 2 \\
6 & 5 & 4 & 3 & 2 & $(1)$ \\
\hline
\end{tabular}

9)

\begin{tabular}{|ccc|ccc|}
\hline (1) & 3 & 5 & 2 & 6 & 4 \\
3 & (1) & 2 & 4 & 5 & 6 \\
5 & 2 & $(1)$ & 6 & 4 & 3 \\
\hline 2 & 6 & 4 & $(1)$ & 3 & 5 \\
4 & 5 & 6 & 3 & (1) & 2 \\
6 & 4 & 3 & 5 & 2 & (1) \\
\hline
\end{tabular}

12)

\begin{tabular}{|cccccc|}
\hline (1) & 3 & (4) & (2) & 6 & $(5)$ \\
3 & 4 & 6 & $(5)$ & 1 & $(2)$ \\
$(4)$ & 5 & $(1)$ & 3 & 2 & 6 \\
$(2)$ & 66 & 3 & 4 & 5 & 1 \\
5 & 1 & 2 & 6 & $(3)$ & $(4)$ \\
(6) & $(2)$ & 5 & 1 & $(4)$ & $(3)$ \\
\hline
\end{tabular}

\section{Reflections}

Let $\mathrm{M}$ be a MOL(6) given as a linear space on $R \cup C \cup D \cup T$, and let $\mathrm{L}$ the sub-LQ(6) defined by the linear space on $R \cup C \cup D$, then:

Theorem 1 Each collineation of L extends uniquely to a collineation of $M$, mapping $T$ to itself. Conversely, each collineation of $M$, which maps $T$ to $T$, induces a collineation of $L$.

Proof Each 3-block of L extends uniquely to a 4-block of M. Each collineation $\varphi$ of L maps each transversal of $L$ to another transversal, and each disjoint pair of transversals to a disjoint pair. Therefore $\varphi$ induces a bijection of $\mathrm{T}$ and hence a bijection of the point set $R \cup C \cup D \cup T$ of M. Since $\varphi$ is a collineation of $\mathrm{L}$ and maps 3-blocks of $\mathrm{L}$ to 3-blocks of L, and since each 3-block extends uniquely to a 4-block of $\mathrm{M}$, it follows 


\begin{tabular}{llllllll}
\hline No. & $\left(\mu_{3}, \lambda, \tau\right)$ & $|A|$ & $|I|$ & $A / I$ & Permutations & $T a$ & $F i-Y a$ \\
\hline 1 & $(9,27,0)$ & 1296 & 216 & $S_{3}$ & {$\left[3^{6} 2^{9}\right]^{3}$} & 1 & 17 \\
2 & $(9,9,0)$ & 432 & 72 & $S_{3}$ & {$\left[6^{6} 3^{6} 2^{3}\right]^{3}$} & 7 & 13 \\
3 & $(9,9,0)$ & 72 & 36 & $C_{2}$ & {$\left[4^{9} 3^{6}\right]^{2}\left[6^{6} 3^{6} 2^{3}\right]$} & 6,12 & 11,12 \\
4 & $(9,0,0)$ & 648 & 108 & $S_{3}$ & {$\left[3^{6} 6^{9}\right]^{3}$} & 16 & 14 \\
5 & $(8,19,0)$ & 48 & 8 & $S_{3}$ & {$\left[6^{4} 4^{4} 3^{2} 2^{5}\right]^{3}$} & 2 & 7 \\
6 & $(8,9,32)$ & 144 & 24 & $S_{3}$ & {$\left[6^{6} 3^{6} 2^{3}\right]^{3}$} & 7 bis & 10 \\
7 & $(8,5,8)$ & 8 & 4 & $C_{2}$ & {$\left[6^{8} 4^{5} 3^{2}\right]^{2}\left[6^{8} 4^{2} 3^{4} 2\right]$} & 10,14 & 1,2 \\
8 & $(7,15,24)$ & 240 & 120 & $C_{2}$ & {$\left[4^{15}\right]^{2}\left[3^{10} 2^{5}\right]$} & 3,11 & 15,16 \\
9 & $(7,15,0)$ & 24 & 12 & $C_{2}$ & {$\left[6^{6} 4^{6} 2^{3}\right]^{2}\left[6^{2} 4^{6} 3^{4} 2^{3}\right]$} & 4,5 & 8,9 \\
10 & $(7,11,8)$ & 24 & 4 & $S_{3}$ & {$\left[6^{4} 4^{8} 3^{2} 2\right]^{3}$} & 8 & 4 \\
11 & $(7,7,8)$ & 16 & 8 & $C_{2}$ & {$\left[6^{8} 4^{7}\right]^{2}\left[6^{4} 4^{4} 3^{6} 2\right]$} & 9,13 & 5,6 \\
12 & $(7,4,8)$ & 24 & 4 & $S_{3}$ & {$\left[6^{9} 4^{4} 3^{2}\right]^{3}$} & 15 & 3 \\
\hline
\end{tabular}

that the extension of $\varphi$ maps 4-blocks of $\mathrm{M}$ to 4-blocks of $\mathrm{M}$. This means that the extension of $\varphi$ is a collineation of $\mathrm{M}$ mapping $\mathrm{T}$ to $\mathrm{T}$.

If, conversely, a collineation of $\mathrm{M}$ maps $\mathrm{T}$ to $\mathrm{T}$, then it maps also $R \cup C \cup D$ to $R \cup C \cup D$ and restricts therefore to a collineation of $\mathrm{L}$.

Theorem 2 Let L be a latin square of order 6 which admits a collineation $\sigma$ with the property: $\sigma$ fixes the main diagonal $H$ elementwise and defines a reflection with respect this diagonal. Then L has no orthogonal latin square.

Proof We suppose, by contradiction, that there exists an orthogonal square, in other words, that L has 6 pairwise disjoint transversals. So we have a MOL(6) and by Theorem 1 the collineation $\sigma$ is also a collineation of this $\operatorname{MOL}(6)$.

(a) There exists no transversal $\mathrm{S}$ which intersects the diagonal $\mathrm{H}$ in exactly one element. Proof: Since this element is fixed under $\sigma$, and since there is exactly one transversal through this element, the transversal $\mathrm{S}$ is invariant under $\sigma$. There are 5 points of $\mathrm{S}$ in the complement of $\mathrm{H}$ and this set is also invariant under $\sigma$. But this set cannot be split into an equal number of points left of $\mathrm{H}$ and right of $\mathrm{T}$ since 5 is odd, a contradiction.

(b) The action of $\sigma$ on the set $\mathrm{D}$ of 6 digits has at most two transpositions. Proof: Assume there are 3 transpositions, then none of the 6 digits can occur on $\mathrm{H}$, since the elements of $\mathrm{H}$ are fixed, a contradiction.

(c) There exists no transversal $\mathrm{S}$ which is invariant under $\sigma$ and disjoint to $\mathrm{H}$. 
Proof: Assume there is an invariant transversal $\mathrm{S}$ disjoint to $\mathrm{H}$. Since $\sigma$ is a reflection with respect to $H$, the 6 disjoint digit values of $\mathrm{S}$ distribute as follows: There are 3 values left side of $\mathrm{H}$ (say 1, 2, 3) and three values right side of $\mathrm{H}$ (say 4, 5, 6). Therefore the reflection $\sigma$ defines three transpositions, a contradiction to b).

Since by (a) no transversal intersects $\mathrm{H}$ in one element, all intersections of transversals with $\mathrm{H}$ have at least 2 elements. Therefore the intersection lengths which partition $\mathrm{H}$ are $(2+4)$ or $(2+2+2)$ or $(3+3)$ or $(6)$.

Case $(2+4)$ : If the digits on $\mathrm{H}$ are, say, 1,1 and 2, 2, 2, 2, then a transversal through a digit 1 , which intersects $H$ not in 1 alone intersects $H$ in a point 2 . The transversal through the second 1 intersects in a second point 2 . There remain two points with digit 2. A transversal through one of them cannot contain the second, therefore it intersects $\mathrm{H}$ in only this point. Now we can apply (a) and have a contradiction.

Case $(2+2+2)$ : Let the digits be $1,1,2,2,3,3$, then they have the transversals (second number) $11,12,21,23,32,33$, so $\mathrm{H}$ is met by three transversals. In the complement of $\mathrm{H}$ are three transversals, and since $\sigma$ has order 2, one of these transversals is invariant. This is a contradiction to (c).

Case $(3+3)$ : The digits 1, 1, 1, 2, 2, 2 get the transversals 11, 12, 13, 21, 22, 23 and we get again 3 transversals in the complement of $\mathrm{H}$, a contradiction to (c).

Case (6): Here the 6 digits 1, 1, 1, 1, 1, 1 get the transversals 11, 12, 13, 14, 15, 16 and this is a contradiction to (a). So in all cases we get a contradiction and the theorem is proved.

\section{Theorem 3 For the latin squares no 1-no 12 there exists no orthogonal square.}

Proof One easily checks, using the list, that each of the 12 squares has a reflection with respect to the main diagonal $\mathrm{H}$. Therefore by Theorem 2 there exists no orthogonal square.

\section{Corollary 1 There exists no $\operatorname{MOL}(6)$.}

Funding Open Access funding enabled and organized by Projekt DEAL.

Open Access This article is licensed under a Creative Commons Attribution 4.0 International License, which permits use, sharing, adaptation, distribution and reproduction in any medium or format, as long as you give appropriate credit to the original author(s) and the source, provide a link to the Creative Commons licence, and indicate if changes were made. The images or other third party material in this article are included in the article's Creative Commons licence, unless indicated otherwise in a credit line to the material. If material is not included in the article's Creative Commons licence and your intended use is not permitted by statutory regulation or exceeds the permitted use, you will need to obtain permission directly from the copyright holder. To view a copy of this licence, visit http://creativecommons.org/licenses/by/4.0/.

\section{References}

Beth, Th., Jungnickel, D., Lenz, H.: Design Theory, Bibl. Inst. Mannheim, Wien, Zürich (1985)

Betten, A., Betten, D.: Proper Linear Spaces on 18 Points. In: Proc. Eur. Conf. Alg. Comb. Appl., ed. A. Betten et al. Gössweinstein, 1999, Springer, pp. 40-54 (2001)

Betten, D.: Zum Satz von Euler-Tarry. Der Math. Naturwissenschaftliche Unterricht 36, 449-453 (1983)

Betten, D.: Die 12 lateinischen Quadrate der Ordnung 6. Mitteilungen Math. Sem. Giessen 136, 181-188 (1984) 
Betten, D.: The Reye configuration in a MOL(6). Mitt. Math. Ges. Hamburg 39, 59-76 (2019)

Betten, D., Braun, M.: A tactical decomposition for incidence structures. Ann. Discr. Math. 52, 37-43 (1992)

Colbourne, C.J., Dinitz, J.H.: The CRC Handbook of Combinatorial Designs. CRC Press, Boca Raton, New York, London, Tokyo (1996)

Denes, J., Keedwell, A.D.: Latin Squares and Their Applications. English University Press, London (1974)

Euler, L.: Recherches sur une nouvelle espece des quarres magiques, Leonardi Euleri opera, ser. I, vol. 7, pp. 291-392, Leipzig, Berlin: Teubner, 1923 (1782)

Fisher, R.A., Yates, F.: The 6x6 Latin squares. Proc. Cambridge Phil. Soc. 30, 492-507 (1934)

Stinson, D.R.: A short proof of the Nonexistence of a Pair of Orthogonal Latin Squares of Order Six. J. Comb. Theory A 36, 373-376 (1984)

Tarry, M.G.: Le probleme des 36 officiers. Comptes Rend. Ass. Franc. Sci. Nat. 2, 170-203 (1901). [1 (1900) ]

Publisher's Note Springer Nature remains neutral with regard to jurisdictional claims in published maps and institutional affiliations. 